\title{
Privatisierung von Sicherheit - Zur Problematik des Outsourcings militärischer Dienstleistungen
}

Private Militärfirmen und „neue Söldner“ sind ein wenig bekanntes Phänomen. Das private Kriegsgeschäft ist seit dem Ende des Kalten Krieges und der Zersplitterung der Interessensphären zu einer Wachstumsbranche geworden, die nicht nur Riesengewinne verbucht, sondern auch die Weltpolitik nachhaltig verändert. Private haben inzwischen Aufgaben übernommen, die einst in staatliche Hoheit fielen. Die Auslagerung militärischer Aufgaben bedeutet Privatisierung der äußeren Sicherheit. Die Aushöhlung des staatlichen Gewaltmonopols, das Entstehen rechtsfreier Räume und die Aushebelung des Völkerrechts sind dabei nur einige Gefahren, die als Folge des Outsourcings militärischer Dienstleistungen entstehen. Staatliche Regelungen für diese neue Dienstleistungsbranche sind dringend vonnöten. \section{Private Militärfirmen -
Überblick und Problemstellung}

Gegenwärtig sind über den Globus mehr als eine Million Angestellte von privaten Militärfirmen - darunter auch viele deutsche Staatsbürger - verstreut. Die Anzahl der privaten Militärfirmen (PMF), bei denen sie angestellt sind, beläuft sich auf über Tausend. Im vergangenen Jahr verzeichnete die Branche einen Umsatz, der 200 Mrd. US-Dollar überstieg. Von allen Wirtschaftszweigen hatte sie die höchsten Wachstums- und Profitraten.

Die privaten Militärfirmen decken den gesamten Aufgabenkatalog ab, der hinsichtlich der äußeren Sicherheit den nationalen Armeen übertragen ist. Und viele dieser Unternehmen nehmen als private Sicherheitsfirmen nach Innen Aufgaben der Polizei und anderer Ordnungskräfte wahr. ${ }^{1}$ Sie übernehmen damit Kernaufgaben des Staates, die im weitesten Sinne unter das Gewaltmonopol fallen (Eppler 2002). Bezogen auf militärische Aufgaben heißt das: Kampfaufträge, Logistik (einschließlich Nachschub und Versorgung) sowie Ausbildung und Beratung. Darüber hinaus haben sich die PMF fest im Intelligence-Sektor etabliert.

Der rasante Aufstieg dieser neuen Dienstleistungsbranche hängt vor allem mit drei Punkten zusammen. ${ }^{2}$ Einerseits erzeugte die neue „Weltsicherheits-Unordnung“, die sich nach Ende der Bipolarität und mit dem Wegfall der von den Supermächten gewährten Sicherheitsschirme entwickelte, zunehmend auf allen Kontinenten Grenzstreitigkeiten, ungelöste interne Verteilungskonflikte sowie ethnische und religiöse Auseinandersetzungen. Mit weniger Sicherheit auf sich allein gestellt und mit weniger Geld für die Staatshaushalte schlitterten viele Staaten der Dritten Welt allmählich in die Katastrophe: Wachsender Niedergang des Nationalstaats, Erosion des Gewaltmonopols, schwindende Souveränität des Staates, allmähliche Auflösung des Gemeinwesens und Bildung von gesellschaftlichen Teilgruppen - dies und anderes sind Erscheinungen derselben Entwicklung, die sich im letzten Jahrzehnt in den "schwachen Staaten“ herauskristallisiert hat (Avant 2005.)

Zum anderen machte sich eine Transformation der Kriegsführung entscheidend bemerkbar, die in Konzepten wie der „Revolution in Military Affairs"(RMA) und „Network Centric Warfare"(NCW) ihren Niederschlag fand. Im Mittelpunkt steht dabei die durch den Einsatz von Elektronik und Informationstechnologien eingeleitete Umwälzung zum einen in der Waffentechnik und zum anderen in der Technik der Kriegsführung. Die Rüstungsgüterindustrie hatte die Entwicklung so weit getrieben, dass ohne elektronische Systeme und IT-Vernetzung keine Kriegsgeräte außer leichten Waffen mehr einsetzbar sind (Metz 2002).

Eine ähnliche Entwicklung wie im militärindustriellen Komplex hat sich im Bereich der Nachrichten-, Informations- und Geheimdienste vollzogen. In diesem wo- möglich noch sensibleren Bereich, was die Sicherheit eines Landes angeht, hat sich die „elektronische Revolution" noch viel entscheidender bemerkbar gemacht. Das betrifft in erster Linie die Computerisierung der nachrichtendienstlichen Mittel und Methoden, die eine Umwälzung der Arbeitsweise nach sich zog. Das betrifft in zweiter Linie die stetig gesteigerte Abhängigkeit der Geheimdienste von den Technologien und dem Know-how der Privatindustrie. Dadurch hat sich drittens eine Verflechtung zwischen Öffentlichem und Privatem ergeben, die womöglich noch größer ist als die im - engsten Sinne - militärischen Sektor.

Betrachtet man die Verteilung der angestellten „Söldner“ über den Erdball, so konzentrieren sie sich vor allem auf die Sicherung von drei Bereichen: erstens von für die westliche Welt wichtigen Erdöl-, Erdgas- und Rohstoffgebieten; zweitens sollen Transportwege für diese Primärgüter und

\footnotetext{
Im Folgenden wird auf die Problematik der inneren Sicherheit nicht näher eingegangen.

2 Zu den Rahmenbedingungen der Expansion der PMF vgl. ausführlich Uesseler 2006.
}

Rolf Uesseler, freier Publizist und Wissenschaftler in Rom. Arbeitsschwerpunkte: Illegale Trends in der Weltwirtschaft, Schattenökonomie, organisierte Kriminalität und Gewaltmärkte; Konfliktbewältigung, Sicherheit und Entdemokratisierung. e-mail: rolfuesseler@hotmail.com 
drittens billige Produktionszonen („Export Processing Zones" - EPZ) gesichert werden. Energiesicherung ist das Haupteinsatzfeld der privaten Militärfirmen auf fünf Kontinenten. Dazu gehört die militärische Absicherung der Fördergebiete, die Sicherung der Pipelines (einschließlich der Länder, durch die sie verlaufen), die Protektion der Seewege sowie der Häfen und Tanker, d.h. die Eskortierung des Öls von der Extraktion bis zur Lieferung in das Verbraucherland.

Ziel des Beitrages ist es, die Probleme aufzuzeigen, die entstehen, wenn die hoheitlichen Aufgaben äußerer Sicherheit privatisiert werden (Abschnitt 4). Dafür werden die Strukturen, Aktionsbereiche und Akteure der neuen militärischen Dienstleistungsbranche zunächst dargestellt (Abschnitte 2 und 3). Abschließend werden Vorschläge formuliert, wie mit Hilfe nationalen und internationalen Rechts die Branche militärischer Dienstleistungen im Sinne der Sicherheit von Staaten, Bürgern und der in diesem Bereich Beschäftigten reguliert werden kann (Abschnitt 5).

\section{Die neue militärische Dienstleistungsbranche}

Die privaten Militärfirmen sind in erster Linie in den hoch industrialisierten Ländern beheimatet - vor allem in den USA und Großbritannien. In der Bundesrepublik sind fast alle großen privaten Militärfirmen mit Zweigniederlassungen vertreten. Sie rekrutieren dementsprechend auch deutsches Personal für alle Sparten (also nicht nur als body-guards). Firmen mit rein deutschem Kapital gehören bisher zu den kleineren Anbietern von militärischen Dienstleistungen. Die Branche wächst jedoch rasant. Und mit der im Gang befindlichen Privatisierung von Teilen der Bundeswehr und dem Wirken der Gesellschaft für Entwicklung, Beschaffung und Betrieb $\mathrm{GmbH}$ (g.e.b.b.), der ein Teil der Umsetzung dieses Vorhabens obliegt, werden auch deutsche Firmen zu den Großen dieser Branche aufsteigen (dazu ausführlich Petersohn 2006).

Die größten Firmen findet man mit ihren Mutterhäusern unter den ersten Hundert der am höchsten dotierten Aktiengesellschaften ihrer jeweiligen Länder. Die ausgewiesenen Umsätze der Marktfüh- rer unter den PMF liegen zwischen 1 und 6 Mrd. €. Die Mehrzahl der PMF bewegt sich mit ihren Einnahmen unter der Milliarden-Euro-Grenze.

Einige Militärfirmen verfügen über mehr Generäle im Aufsichtsrat, als in den Armeen ihrer jeweiligen Heimatländer (USA oder GB) gerade Dienst tun; andere über mehr Analysten, Computerspezialisten und Nachrichtendienstler, als Geheimdiensten ganzer Länder zur Verfügung stehen. Der größte Rüstungskonzern der Welt, Lokheed-Martin, mit seinen zahlreichen PMF schreibt beispielsweise mehr Software Programme als selbst Microsoft. Der Standard an Spezialwissen und speziellen Fertigkeiten ist in vielen PMF so hoch, dass reguläre Armeen nach ihren eigenen Aussagen diese Qualitätsstufe gar nicht mehr erreichen können (Schreier/Caparini 2005).

Die PMF handeln aus einer betriebswirtschaftlichen Perspektive, wenn auch politische Überlegungen gelegentlich eine Rolle spielen. Folglich ist ihr Handeln primär darauf gerichtet, die Kosten zu minimieren und die Profite zu maximieren, und allenfalls sekundär darauf, einen bestimmten Qualitätsstandard von Sicherheit zu erreichen.

Ihre Firmenphilosophie verkaufen die PMF offensiv nach außen als von politischer Ethik getragene Friedensmissionen, als unermüdlichen Einsatz zur Beendigung von Kriegen und Konflikten. Den Rest an Marketing besorgt eine der einflussreichsten Lobbygruppierungen der Welt, die International Peace Operations Association (IPOA).

In Führungsstil und Management unterscheiden sich die PMF entscheidend von den alten Söldnertrupps, aber kaum von Unternehmen in anderen Branchen. Sie gestalten und lenken ihre Betriebe wie komplexe Systeme, steuern sie nach den neuesten Wirtschafts- und Marketingtheorien, vertrauen auf ein starkes Lobbying bei der Akquirierung von Aufträgen und treffen ihre Entscheidungen immer mit Blick auf den „shareholder-value“.

Die Größe der privaten Militärfirmen variiert enorm - von Ein-Mann-Betrieben bis zu Großkonzernen mit mehreren zehntausend Angestellten. Dementsprechend ändern sich die Rechtsformen von Personengesellschaften über $\mathrm{GmbHs}$ bis zu Aktiengesellschaften. Viele der erfolgreichsten Militärfirmen sind inzwischen Teil von weitverzweigten Holdings oder von Rüstungsgiganten geworden. Die Aufkäufe und Fusionen erfolgten zum beiderseitigen Vorteil. Die „Mutterhäuser“ erweiterten ihre Angebotspalette um den immer wichtiger werdenden Bereich der militärischen und sicherheitstechnischen Dienstleistungen, die PMF gewannen Zugang zu nationalen wie internationalen Kapital- und Finanzmärkten (Singer 2003).

Die Herkunftsländer der überwiegenden Anzahl von PMF liegen in Nordamerika und Europa, wenn auch in den Staaten der Dritten Welt das Outsourcing in diesem Bereich enorm gestiegen ist.

Ein Großteil der privaten Militärfirmen gehört heute zu den "global player": Sie arbeiten für verschiedene Auftraggeber oder Regierungen, ihre Standorte haben sie über den Globus verteilt und ihre Einsätze finden auf allen Kontinenten statt.

Die PMF sind außer durch zivilrechtliche Verträge, die sie mit dem Auftraggeber abschließen, durch nichts gebunden. Diese Handlungsfreiheit ermöglicht es ihnen, auf dem Weltmarkt kundenspezifische Produkte und auftraggeberzentrierte Lösungen $\mathrm{zu}$ entwickeln und anzubieten. Ihre Dienstleistungen sind sozusagen „Wegwerfprodukte": einmal benutzt, sind sie verbraucht; der nächste Kunde bekommt ein anderes „maßgeschneidertes“ Produkt angeboten. Diese Kundenzentriertheit u.a. macht die PMF für die Auftraggeber so attraktiv.

Das von den PMF engagierte Personal ist so bunt zusammengesetzt, wie die Einsatzgebiete unterschiedlich sind. Man trifft alle Volksgruppen und Hautfarben, alle Altersstufen, fast alle Nationalitäten und Angehörige nahezu aller sozialen Schichten. Zwar sind die Militärfirmen für ihre hervorragenden Spezialisten bekannt, aber ebenso für ihr brutales „Lohndumping“. Da sie vor allem auftragsgebunden arbeiten, können sie ihre Unternehmen mit einer relativ kleinen Kernbelegschaft aus Fachkräften betreiben. Die Hauptarbeit erledigen "freie Mitarbeiter“. Manche PMF werben sogar damit, dass sie über 50.000 „free-lancers“ in ihrer Kartei haben. Die gezahlten Löhne variieren zwischen 10 und 1000 US-Dollar pro Tag; aus Gründen der Flexibilität (und der Mortalitätsrate) werden Monatslöhne, außer für die Kernbelegschaft, kaum vereinbart (Holmquist 2005).

Um Lohnkosten zu senken, rekrutieren die PMF den größten Teil der „Privatsoldaten" am Ort ihrer Einsatzgebiete. Das Verhältnis zwischen „hoch spezialisierter 
Fachkraft“ und einheimischen „Sicherheitskräften“ beträgt nicht selten eins zu zwanzig oder mehr. Da die Arbeitslosigkeit in den Ländern der Dritten Welt und im ehemaligen Ostblock auch für Ex-Soldaten oder Polizisten weitaus höher liegt als im Westen, fällt es den PMF nicht schwer, dort billiges Personal zu finden. Doch nicht nur um des Lohndumpings willen greifen die PMF auf Personal vor Ort zurück. Dieses garantiert wegen seiner Sprachkenntnisse und seiner Vertrautheit mit den heimischen Gewohnheiten auch ein leichteres und tieferes Eindringen in die Sozial- und Infrastrukturen der Konfliktgebiete, in denen sie ihren Auftrag erledigen.

Die Erwerbslosigkeit von Millionen Soldaten nach dem Ende des Ost-WestKonflikts hat der militärischen Dienstleistungsbranche einen Arbeitsmarkt beschert, von dem die übrige Wirtschaft träumt. Selbst für delikate Aufgaben und riskante Einsätze finden sie jederzeit frei verfügbares, hoch qualifiziertes Personal, und das zu extrem günstigen Preisen und Bedingungen. Lohnnebenkosten, Tariflöhne, Gewerkschaften, Aufsichtsämter, Kontrollbehörden - mit all dem muss sich die Branche nicht auseinandersetzen.

\section{Die Auftraggeber}

Die militärische Dienstleistungsbranche hätte nicht so expandieren und sich mit ihren Angeboten so diversifizieren können, wenn die Nachfrage nicht auch rasant gestiegen wäre.

Den weltweit operierenden Industrieunternehmen oder Versicherungen versprechen die PMF eine Risikobegrenzung und damit verminderte Ausgaben. Staaten und internationalen Organisationen offerieren sie, Sicherheitsaufgaben gleicher Qualität bei geringeren Kosten zu übernehmen. Humanitären Vereinigungen bieten sie an, den Schutz zu erhöhen und die Todes-, Verwundeten- und Kidnappingrate zu senken. Die Angebote sind verlockend, und so stiegen nach und nach auch wegen vermeintlich fehlender Alternativen - immer mehr Auftraggeber in diesen Markt ein.

Die Nachfrageseite für private militärische Dienstleistungen ist extrem uneinheitlich und buntscheckig. Betrachtet man sie auf einer moralischen Skala, so reicht das Spektrum von brutalen Kriegsfürsten und Diktatoren über Rebellengruppen und skrupellose Drogenkartelle bis hin zu souveränen Staaten, angesehenen Wirtschaftsunternehmen und Menschenrechtsorganisationen.

Ordnet man sie nach dem Volumen, so sind die größten Auftraggeber gegenwärtig die sogenannten „globalen“ und „starken“ Staaten. (Im Irak beispielsweise haben die USA inzwischen mehr Angestellte von PMF beschäftigt als eigene Soldaten stationiert.) Der inzwischen zweitgrößte Auftraggeber ist die Privatwirtschaft, wobei es nicht nur "global player", sondern auch mittelgroße Unternehmen sind, die die Dienstleistungen der PMF in Anspruch nehmen. Die dritte Gruppe bilden die „schwachen“ und „verfallenden“ Staaten, vornehmlich aus Konfliktregionen. An vierter Stelle liegt die Gruppe, die aus Bürgerkriegsparteien, terroristischen Netzwerken, Befreiungsbewegungen und kriminellen Organisationen besteht. Zunehmend mehr Aufträge vergeben internationale und transnationale Institutionen oder Bündnisse. Zusammen mit den unzähligen Nicht-Regierungs-Organisationen (NRO) stellen sie die fünfte Gruppe dar. Private Vereinigungen und zivile Einzelpersonen, die sich mit Hilfe von PMF beispielsweise gegen Entführung und bewaffnete Gewalt absichern wollen, bilden die kleinste Gruppe auf der Nachfrageseite.

Was die Privatwirtschaft betrifft, so war sie eine der Ersten, die sich ihre Interessen weltweit von PMF absichern ließ. Sie engagierte die „Neuen Söldner" für Aufgaben selbst in den entlegensten Teilen dieser Erde. Für sie bedeutete es einen besonderen Vorteil, staatliche oder internationale Institutionen nicht erst um Schutz ihres Eigentums und ihrer Aktivitäten angehen zu müssen, sondern ihn direkt bei Privaten anfordern zu können. So beauftragte der Diamanten-Multi De Beers schon Anfang der 1990er Jahre die PMF Executive Outcomes, verdeckte Operationen hinsichtlich Diamantenvorkommen im südlichen Afrika, vor allem in Botswana, Namibia und Angola, für ihn durchzuführen. Bei der Auswahl der geeignetsten Methoden und Mittel, um Sicherheit und ihre ökonomischen Ziele zu erreichen, ließ die Privatwirtschaft den PMF weitgehend freie Hand. Gewaltanwendung und Missachtung humanitärer Prinzipien waren dabei jedoch so zahlreich und teilweise so eklatant, dass sie den Protest der Medien her- vorriefen. Die Berichte von Menschenrechtsorganisationen (wie Human Rights Watch oder Amnesty International), von Gewerkschaften oder von der International Labour Organisation (ILO) der UN über die illegalen Praktiken der PMF, die im Auftrag oder mit Billigung der Unternehmen handelten, füllten bald Regale (Pagliani 2004). Die wiederholten und langjährigen Kritiken an dieser Art der Zusammenarbeit von Privatwirtschaft und privaten Militärfirmen führte dazu, dass sich im Juli 2000 einige transnationale Konzerne (TNK) aus aller Welt unter der Schirmherrschaft der UNO zur „Global Compact“ zusammenfanden, um allgemeine Verhaltensregeln zu entwerfen. Bisher konnte allerdings noch nicht festgestellt werden, dass mit dieser Absichtserklärung einiger "global player" und ihrem Akt der Selbstregulierung eine Verbesserung im Verhalten der Wirtschaft allgemein eingetreten ist.

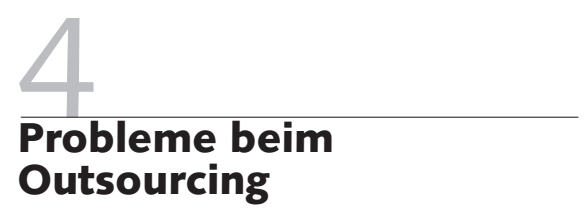

\subsection{VERTRAGSPROBLEME}

Die Schwierigkeiten beginnen mit der Auftragsvergabe. Nur in den seltensten Fällen - wie die Praxis zeigt - ist das Anfangsmandat so detailliert beschrieben und aufgeschlüsselt, dass daraus rechtliche Ansprüche des Auftraggebers abgeleitet werden könnten. Noch weniger erfährt man darüber, welche Sanktionen im Falle einer Verletzung oder Nichterfüllung des Vertrages vorgesehen sind. Das heißt, Selbstverständlichkeiten, die selbst zu einem simplen Kaufvertrag gehören, werden in einem so sensiblen Bereich wie dem der Anwendung von bewaffneter Gewalt mit staatlicher Duldung außer Acht gelassen. Im Allgemeinen wird auch nicht spezifiziert, wie und mit welchen Mitteln - und mit welchen nicht - ein Auftrag auszuführen ist. Angestellte von PMF im Irak haben beispielsweise darauf hingewiesen, dass sie - ohne besondere Genehmigungen einholen zu müssen - befugt seien, Leute zu verhaften, Straßensperren zu errichten, Ausweise zu konfiszieren etc. Niemand konnte klären, ob es diesen PMF tatsächlich mit Rückendeckung des Pentagon vertraglich gestattet wurde, sich hoheitliche 
Aufgaben zu eigen zu machen (Isenberg 2004.)

Die Vertragsprobleme beschränken sich aber nicht nur auf die „Kontrolle der Ausübung von Gewalt". In der Mehrheit der Fälle handelt es sich um eine Schlechterfüllung der Aufträge. Da vor allem in den angelsächsischen Ländern inzwischen der Glaube „Privat ist billiger als Staat“ genügt, um Aufträge auch bei mangelnden konkurrierenden Angeboten und ohne kontrollierte Ausschreibung zu vergeben, ist es nicht verwunderlich, dass ein Instrument wie die Qualitätskontrolle kaum einmal angewendet wird. Erst wenn sich beispielsweise - wie geschehen - das reguläre Heer über die logistischen Leistungen der PMF beschwert, wird nachgefragt, was die Verträge für Leistungen vorsehen (Holmquist 2005). In den vergangenen zehn Jahren war es nicht die Ausnahme, sondern fast die Regel, dass das Preis-Leistungs-Verhältnis bei den PMF so aussah: überhöhte Preise für minderwertige Leistungen und die Qualitätskontrolle lag beim Endverbraucher. Hinzu kommt, dass mit dem Argument des "schlanken Staates" die Privatisierungsbefürworter auch erfolgreich bei der Einsparung staatlichen Kontrollpersonals waren. So hat sich beispielsweise in den USA von 1997 bis 2005 das Auftragsvolumen für die PMF verdoppelt, die für die Ausarbeitung, den Abschluss und die Überwachung von Verträgen zuständigen Angestellten sind jedoch um ein Drittel reduziert worden. Damit hat sich auch im militärischen Bereich die Abneigung der Privatwirtschaft gegen Regulierung und staatliche Kontrollen durchgesetzt; und auf politischer Seite folgt man dieser Auffassung, zumindest durch Unterlassung, indem man keine Ressourcen für Überprüfungen zur Verfügung stellt (Wulf 2005).

Nach zahlreichen Untersuchungen über die PMF fasst das Fachblatt der Logistik-Truppen in den US-Streitkräften die langjährigen Erfahrungen in sieben Punkten zusammen. Kritisiert wird:

- das Fehlen einer Einsatzdoktrin über die Verantwortlichkeit in der Kommunikation; - der Verlust des Überblicks über die auf dem Gefechtsfeld verfügbaren Waffen und andere militärische Geräte;

- der Verlust von Kontrolle über Kontraktpersonal und Gerät;

- eine gestiegene Verantwortung der Streitkräfte für die Sicherheit des Kontraktpersonals;
- der Bedarf an zusätzlichem (!) Militärpersonal, Material und Finanzen, um das Kontraktpersonal zu unterstützen;

- die unzureichende Verlässlichkeit der Kontraktfirmen bei Lieferungen in eine feindliche Umgebung;

- das Problem merklicher Lücken im Nachschub, wenn die kommerziellen Lieferwege unterbrochen werden (Uesseler 2006).

So ist es bisher nicht mehr als eine Annahme, nicht mehr als ein ideologisch untermauerter Glaube, dass Privatisierung im Militärsektor billiger sei. Die von der Privatwirtschaft in die Öffentlichkeit gestreuten Erfolgsstatistiken sind beispielsweise noch nie einer Qualitätskontrolle unterzogen worden, und eine solide, auf betriebswirtschaftlicher Kostenrechnung basierende Untersuchung hat die interessierte Seite bisher nicht vorlegen können. Wissenschaftliche Untersuchungen wiederum haben keinen Beweis für die Richtigkeit der Behauptung, „privat ist billiger“, erbracht. Alle verfügbaren Zahlen weisen im Gegenteil darauf hin, dass die Auslagerung von militärischen Dienstleistungen in die Privatwirtschaft den Steuerzahler teurer kommt (Wulf 2005).

Ein weit gravierenderes Problem, das auch durch noch so gut ausgearbeitete und überwachte Verträge nicht aus der Welt geschafft werden kann, hängt mit der Vertragsstruktur selbst zusammen. PMF sind geschäftlich motivierte Akteure, die sich in den seltensten Fällen für gesellschaftliche Ziele und deren Verwirklichung interessieren. Mit der Privatwirtschaft Verträge abschließen, heißt, sich der Tatsache bewusst zu sein, dass diese aus der vereinbarten Verpflichtung aussteigen kann, wann immer sie es aus Eigennutz für opportun hält. Wenn einer Firma im Laufe der Vertragsabwicklung die Risiken zu hoch erscheinen oder ihr ein neuer, lukrativerer Auftrag winkt, wird sie ohne Weiteres einseitig den Vertrag auflösen, die vereinbarten Sanktionen und auch die Gefahr in Kauf nehmen, bei der nächsten Ausschreibung nicht berücksichtigt zu werden. Niemand kann eine PMF dazu zwingen, ihren Auftrag durchzuführen. Anders als im Zivilbereich hat dies im militärischen erhebliche Konsequenzen. So ist es in den letzten drei größeren kriegerischen Auseinandersetzungen-Balkan-, Afghanistan-, Irak-Krieg - mehrfach geschehen, dass sowohl einzelne „Privatsoldaten“ wie ganze Militärfirmen ihren Verpflichtungen, beispielsweise zur Versorgung der kämpfenden regulären Truppen, nicht nachgekommen sind. Sie haben sich wegen des Risikos, getötet zu werden, schlicht geweigert, die „regulären Soldaten " an der Front mit Benzin, Wasser, Nahrung, Munition zu beliefern. Als z. B. im Dezember 2003 der Norden Bagdads zur „heißen Zone“ wurde und in den Kämpfen zwei südkoreanische „Söldner“ getötet wurden, verließen am nächsten Tag 60 ihrer Landsleute die Positionen und weigerten sich, ihren Dienst wieder aufzunehmen (Isenberg 2004). Es ist daher nicht verwunderlich, dass sich in den Streitkräften der Unmut über die für Nachschub verantwortlichen PMF regt, weil sie dadurch selbst, ohne eigenes Zutun in eine risikoreiche, wenn nicht gar lebensbedrohliche Lage hineinmanövriert werden. Eine Handhabe, wie sie ihnen intern für Befehlsverweigerung oder Desertion zur Verfügung steht, haben sie gegenüber den PMF als privatem Wirtschaftssubjekt nicht.

\subsection{GESETZLICHE PROBLEME}

Probleme wie Rechenschaftspflicht, Verantwortlichkeit, Kontrolle oder Transparenz bestehen aber nicht nur auf der vertraglichen, sondern auch auf der gesetzlichen Ebene. Wem staatliche Beamte oder Angestellte aus dem Sicherheitssektor (Polizisten, Soldaten etc.) verantwortlich und rechenschaftspflichtig sind, ist rechtlich strengstens geregelt. Für die PMF und ihr Personal gilt dies nicht. Was der Staat als Auftraggeber von den PMF verlangen kann, ist, dass sie sich an international verbindliche Regeln und nationale Gesetze halten. Wie sich die „Privatsoldaten“ vor Ort in einer Konfliktsituation konkret verhalten, ist allein Sache der Firma. Zwar behaupten die Militärfirmen, dass sie ihr Personal instruieren und anweisen würden, Gesetze und Regeln einzuhalten und Menschenrechte zu achten, doch außer dieser Absichtserklärung hat der Staat, wenn er die „neuen Söldner" einsetzt, keinerlei Kontrollmöglichkeit. Selbst wenn ihm Gesetzesverletzungen bekannt werden, sind ihm weitgehend die Hände gebunden. Wie unzählige Beispiele von den Einsatzfeldern der PMF in aller Welt belegen, werden von ihrem Personal immer wieder kriminelle Handlungen und Menschenrechtsverletzungen begangen. Eine gesetzliche Handhabe, gegen diese Individuen strafrechtlich vorzugehen, hat der beauftragende und 
entsendende Staat - anders als bei seinen eigenen Soldaten - faktisch ebenso wenig wie der Staat, auf dessen Hoheitsgebiet diese Verbrechen begangen werden (Schaller 2005).

Es ist vor allem der rechtliche Status der PMF-Angestellten, der die gesetzliche Lage und deren Kontrolle so problemreich macht. Das Völkerrecht (Genfer Convention III, IV von 1949) kennt für den Kriegsfall nur den Kombattanten und die Zivilbevölkerung; auf sie ist der rechtliche Schutz zugeschnitten. Die „neuen Söldner“ sind aber keine „Kombattanten“, weil sie vom Auftrag her nicht zu den kämpfenden Truppen gezählt werden und nicht der militärischen Befehlsgewalt unterstehen. Sie sind aber auch keine „Zivilisten“, weil sie in die Kriegsmaschinerie eingebunden, häufig im Auftrag von Regierungen tätig und nicht selten bewaffnet sind. Aber auch unter die eng gefasste Definition der „Söldner" in der Genfer Konvention (Artikel 47) können die Angestellten der PMF ebenso wenig wie ihre Firmen subsumiert werden. Unter anderem, weil alle dort aufgeführten sieben Merkmale erfüllt sein müssen und weil in der Regel ein am Konflikt beteiligter Staat die „neuen Söldner“ beauftragt hat (Schaller 2005).

\subsection{POLITISCHE PROBLEME}

Das nahezu unkontrollierte Wirken der privaten Militärfirmen und ihrer Angestellten wirft nicht nur Probleme in den „Krisengebieten“ auf, in denen sie tätig sind, sondern hat auch Rückwirkungen kurz-, mittel- und langfristiger Art auf die auftraggebenden Länder selbst. Die vielfach praktizierte Folter in (Geheim-)Gefängnissen - zum guten Teil von Personen privater Militärfirmen vorgenommen - ist dafür nur ein Beispiel. Da Art. 47 der Genfer Konvention nicht greift und die Angestellten der PMF sich in einer rechtlichen Grauzone bewegen, ist ihnen Straffreiheit selbst bei Tötungsdelikten so gut wie zugesichert. Die betroffene Seite wehrt sich nicht selten mit Entführungen von Privatpersonen, Erpressungen und terroristischen Drohungen. Es entsteht eine Gewaltspirale.

Die Privatisierung von Sicherheit mit Hilfe privater Militärfirmen verändert aber auch das demokratische Gefüge in den westlichen Ländern selbst. Über LockheedMartin - den größten Rüstungskonzern der Welt, unter dessen Dach sich potente
PMF wie MPRI, TITAN, Sytex oder ACS bewegen -, schrieb die New York Times, dass dieser Koloss Amerika zwar nicht regieren würde, aber ohne oder gegen Lockheed-Martin könne die USA auch nicht mehr regiert werden.

Es ist ein Merkmal demokratischer Staaten, die militärische Gewalt in kontrollierbaren Bahnen zu halten und den Einfluss des Militärs auf Politik und Zivilgesellschaft weitgehend einzugrenzen. Die Privatisierung hat jedoch schon jetzt dazu geführt, dass die PMF direkt oder indirekt einen immer größeren Einfluss auf die staatlichen wie globalen Interventions-, Konflikt- und Kriegsstrategien ausüben. Wie bei Interventionen im Einzelnen vorgegangen wird, welche Mittel eingesetzt werden und welche Gesichtspunkte im Vordergrund stehen, bei all diesen Fragen sprechen PMF inzwischen ein entscheidendes, manchmal das entscheidende Wort mit. Es hängt heute auch schon von den Verdienstmöglichkeiten der privaten Militärfirmen $\mathrm{ab}$, wie eine militärische Aktion durchgeführt wird, welche außenpolitischen Akzente gesetzt werden (Singer 2003). Dieser Druck auf die Politik kommt verstärkt zum Ausdruck, wenn die privatwirtschaftlichen Interessen der militärischen Dienstleistungsbranche sich mit denen aus Industrie und dem Finanzsektor treffen.

\subsection{ZWISCHENFAZIT}

Der Einsatz von privaten Militärfirmen hat in unterschiedlichen Bereichen Probleme aufgeworfen, die bisher nicht zufriedenstellend gelöst worden sind. Vom Standpunkt der privaten militärischen Sicherheitsbranche und vieler Auftraggeber gibt es einige Argumente, die für eine Privatisierung von öffentlichen Leistungen auf dem Gebiet der Sicherheit sprechen. Aus der Sicht der Bürger bzw. der von militärischen Einsätzen betroffenen Bevölkerung, aber auch aus Perspektive der staatlichen Militärs, gibt es mindestens genauso viele Gründe, die dagegen sprechen. Übersicht 1 zeigt die wichtigsten Argumente für und wider die Privatisierung von Sicherheitsleistungen.

Das Dilemma mangelnder Transparenz, Rechenschaftspflicht und Kontrolle ist seit geraumer Zeit bekannt. In den angelsächsischen Ländern hatte man es bereits nach den ersten Erfahrungen thematisiert, also bevor man in Deutschland mit der Privatisierung begann (Leander 2002).
Inzwischen überschlagen sich die Publikationen und mehren sich die Seminare, in denen Regelungen und Modelle, Verordnungen und Gesetze diskutiert werden, wie man der Probleme Herr werden kann. Eine einigermaßen befriedigende Lösung konnte bisher nicht einmal in Ansätzen gefunden werden (Richards/Smith 2007). Der heute herrschende Maßstab für Transparenz, Rechenschaftspflicht und Kontrolle, so wie er beispielsweise für die Bundeswehr, die Polizei, den Zoll, den Bundesgrenzschutz und die verschiedenen Geheimdienste gilt, wird bei der Beauftragung von PMF nicht angelegt.

So agieren die privaten Militärfirmen in einem regelfreien Raum, der an Willkürherrschaft grenzt. Das Demokratiegebot verlangt aber eine zirkuläre Kontrolle. Solange diese für PMF nicht garantiert werden kann, dürften nach den bis heute gültigen Maßstäben keine Aufträge an sie vergeben werden. Aber auch wenn man Regelungen finden würde, die diesem Gebot Genüge täten, würde das Ganze an der ökonomischen Machbarkeit scheitern. Der Apparat, der nach heutigem demokratischen Standard für Transparenz, Rechenschaftspflicht, Kontrolle bei hunderten von privaten Militärfirmen - tätig in hunderttausenden von Orten rund um den Erdball - aufgebaut werden müsste, wäre so groß und folglich auch extrem kostspielig, dass ihn kein Staat bezahlen könnte. Eine demokratischen Regeln gerecht werdende Privatisierung von staatlichen Sicherheitsorganen würde allein an der finanziellen Machbarkeit scheitern.

\section{Schlussfolgerungen}

Welche Folgen sich durch die Privatisierung von Sicherheit in Deutschland für den Staat, die staatlichen Institutionen, für die Gesellschaft und den einzelnen Bürger ergeben, kann gegenwärtig nur weitgehend theoretisch formuliert werden, da dieses Phänomen hier erst in den Anfängen steckt. Aufgrund der Erfahrungen mit PMF in anderen Ländern sind jedoch einige Aussagen über die künftige Entwicklung in Deutschland möglich:

- Die Reduzierung der Kosten für Sicherheitsaufgaben ist durch Auslagerungen nicht zu erwarten; 


\begin{tabular}{|c|c|c|}
\hline \multicolumn{3}{|c|}{ Übersicht 1: Privatisierung von Sicherheit - Pro und Kontra } \\
\hline Bereich & Pro & Kontra \\
\hline Wirtschaftlichkeit & * Firmen arbeiten kostengünstiger & $\begin{array}{l}\text { * Kostensenkung bislang nicht nach- } \\
\text { gewiesen; gegenteilige Evidenz/Erfah- } \\
\text { rung; } \\
\text { * keine Qualitätsprüfung; Preis- } \\
\text { Leistungsverhältnis unklar; } \\
\text { * Firmen sind primär profitorientiert; } \\
\text { * Geschäfte der Firmen sind nicht } \\
\text { transparent; } \\
\text { * Firmen sind nicht rechenschafts- } \\
\text { pflichtig; } \\
\text { * Die wirklichen Kosten der } \\
\text { Militäreinsätze werden verschleiert. }\end{array}$ \\
\hline Militär & $\begin{array}{l}\text { * Militär kann sich auf die } \\
\text { Kernaufgaben konzentrieren; } \\
\text { * Firmen agieren flexibler und } \\
\text { können Personal schneller } \\
\text { entsenden; } \\
\text { * Synergie zwischen Firmen und } \\
\text { Militär. }\end{array}$ & $\begin{array}{l}\text { * Abhängigkeit des Militärs von } \\
\text { Firmen; } \\
\text { * Firmen sind in Kriegssituationen } \\
\text { nicht zuverlässig; } \\
\text { * kurffristige Lagerhaltung bei } \\
\text { Nachschub für Kriegssituationen } \\
\text { ungeeignet; } \\
\text { * fehlende Kooperation zwischen } \\
\text { Militär und Firmen; } \\
\text { * zusätzliche Aufgaben für Militär: } \\
\text { u.a. Schutz des PMF-Personals. }\end{array}$ \\
\hline $\begin{array}{l}\text { Friedenssicherung } \\
\text { (Peacekeeping) und } \\
\text { humanitäre Einsätze }\end{array}$ & $\begin{array}{l}\text { * Rasche Reaktionen der Firmen } \\
\text { in Krisen; } \\
\text { * Qualität und Aktionsradius der } \\
\text { UN-Missionen wird erhöht; } \\
\text { * Schutz für Hilfsorganisationen; } \\
\text { * Einsatz nationaler Truppen- } \\
\text { kontingente kann reduziert werden. }\end{array}$ & $\begin{array}{l}\text { * Schutzverpflichtung von National- } \\
\text { staaten und internationaler Völker- } \\
\text { gemeinschaft wird delegiert und } \\
\text { privatisiert; } \\
\text { * nicht transparente Firmen werden } \\
\text { durch die UN legitimiert; } \\
\text { * Menschenrechtsverletzungen } \\
\text { können nicht aufgedeckt und verfolgt } \\
\text { werden. }\end{array}$ \\
\hline Internationale Krisen & $\begin{array}{l}\text { * Stabilisierung kollabierender } \\
\text { Länder; } \\
\text { * Nutzung des Privatsektors in } \\
\text { Postkonfliktgesellschaften. }\end{array}$ & $\begin{array}{l}\text { * Die Fortsetzung der Kämpfe ist im } \\
\text { Interesse der Firmen; } \\
\text { * Firmen können die Außenpolitik } \\
\text { ihres Heimatlandes diskreditieren; } \\
\text { * die Unterscheidung zwischen } \\
\text { Zivilisten und Soldaten wird verwischt; } \\
\text { * Firmen fungieren als verdeckte } \\
\text { Stellvertreter der Regierung. }\end{array}$ \\
\hline Technologie & $\begin{array}{l}\text { * Firmen verfügen über besseres } \\
\text { technisches Know-how. }\end{array}$ & $\begin{array}{l}\text { * Know-how kann in Kriegssituatio- } \\
\text { nen nicht zur Verfügung stehen oder } \\
\text { verweigert werden; } \\
\text { * Know-how kann missbraucht und } \\
\text { gegen Auftraggeber verwendet } \\
\text { werden. }\end{array}$ \\
\hline Politik & $\begin{array}{l}\text { * Regierungen können durch } \\
\text { Auftragsvergabe an Firmen } \\
\text { Sicherheitsaufgaben auslagern und } \\
\text { die eigenen Streitkräfte flexibler } \\
\text { einsetzen. }\end{array}$ & $\begin{array}{l}\text { * Militärffirmen unterliegen keiner } \\
\text { demokratischen Kontrolle; } \\
\text { * es ist Aufgabe des Staates, } \\
\text { die Sicherheit zu garantieren; } \\
\text { * die schwierige zivil-militärische } \\
\text { Balance wird gestört. }\end{array}$ \\
\hline Recht & $\begin{array}{l}\text { * Firmen arbeiten mit einer Lizenz } \\
\text { von Regierungen; } \\
\text { * Verhaltenskodices können die } \\
\text { rechtmäßigen Handlungen der } \\
\text { Firmen regeln. }\end{array}$ & $\begin{array}{l}\text { * Rechtliche Regeln für Firmenein- } \\
\text { sätze fehlen weitgehend und können } \\
\text { rechtlich nicht überprüft werden; } \\
\text { * Firmen und ihre Angestellten } \\
\text { können gerichtlich kaum für Straf- } \\
\text { rechtsverletzungen belangt werden; } \\
\text { * die Genfer Konvention } \\
\text { (Kombattanten / Nicht-Kombattanten) } \\
\text { wird ausgehebelt. }\end{array}$ \\
\hline & & WSI mitTEILUNGEN \\
\hline
\end{tabular}

- der Privatstatus dieser Unternehmen verhindert effektive parlamentarische Kontrolle und Einflussnahme;

- das staatliche Gewaltmonopol gerät in Gefahr;

- das Primat ziviler Krisenprävention und Krisenbeilegung weicht der militärischen Konfliktlösung.

Die derzeitige Bundesregierung sieht - wie auch schon ihre Vorgängerinnen - in Bezug politischen Handlungsbedarf sieht. Wenn man aus dem bisherigen Schweigen schließt, dass dies nicht der Fall ist, so muss darüber diskutiert werden - in der Öffentlichkeit und in den Medien. Denn einen enormen faktischen Bedarf für politisches Handeln gibt es auch in Deutschland. Es sei nur daran erinnert, dass deutsche Staatsbürger für ausländische PMF tätig sind und für sie schießen, dass deutsche PMF in Konfliktregionen Aufträge abwickeln, dass deutsche Firmen im Ausland PMF beauftragen, dass auf deutschem Boden unzählige ausländische PMF (einschließlich privater Geheimdienste) tätig sind.

Zwei Problempunkte wären vorrangig zu behandeln: Der erste betrifft nationales, der zweite internationales Recht. Im nationalen Rahmen fehlt es an Zulassungs- und Tätigkeitskriterien, Aufgaben- und Kompetenzbereiche für private Militärfirmen sind nicht festgelegt. Die bisherigen Regelungen (u.a. im Gewerberecht) sind nicht zuletzt wegen mangelnder Kontrollmöglichkeiten unzureichend. Ein Bewilligungsverfahren (Lizensierungs- und Registrierungssystem) ist nicht vorgesehen. Eine weitere dringende Herausforderung, die der Einsatz deutscher PMF in Konfliktgebieten aufwirft, ist die fehlende rechtliche und definitorische Regulierung des Rahmens, in dem sie sich bewegen. Es geht um die Spezifizierung der Verantwortlichkeiten dieser Unternehmen und ihrer Angestellten sowie um die Aufsicht und Kontrolle ihrer Aktivitäten. Auftrags- und projektbezogene Genehmigungen analog zum Export von Rüstungsgütern fehlen vollständig. Ähnliches gilt für ausländische private Militärfirmen, die auf deutschem Boden tätig sind.

Auf internationaler Ebene greift gegenwärtig nur das humanitäre Völkerrecht. In der Praxis hat es sich in den letzten zehn Jahren als äußerst schwierig bzw. weitgehend unmöglich erwiesen, die daraus erwachsenden Verantwortlichkeiten auf straf- wie zivilrechtlicher Ebene durchzusetzen. Nur sehr wenige mit Konflikten, Unruhen oder Krisen konfrontierte Staaten regeln die Tätigkeit von PMF auf ihrem Hoheitsgebiet. Da es keine spezifischen völkerrechtlichen Normen für diese Wirtschaftsbranche und ihre Tätigkeiten gibt, sind internationale Normen zu entwickeln und Abkommen zu treffen. Es gilt, auf der Basis des Humanitären Völkerrechts und der Menschenrechte eine Bestärkung und Klärung der völker- 
rechtlichen Pflichten der Staaten und der privaten Militärfirmen (einschließlich ihres Personals) herbeizuführen. Weiterhin wäre eine Ausarbeitung von Empfehlungen und Richtlinien vonnöten, die auf der
Basis der schon bestehenden Verpflichtungen die Staaten bei der Wahrnehmung ihrer Verantwortung für die Durchsetzung des Humanitären Völkerrechts und der Menschenrechte unterstützen. All diese
Maßnahmen wären auf zwischenstaatlicher (inter-governativer), regionaler und UNO-Ebene zu ergreifen und zu implementieren.

\section{LITERATUR}

Avant, D. (2005): The Market of Force. The Consequences of Privatizing Security, Cambridge

Benegas, R. (2001): De la privatisation de la guerre a la privatisation du peacekeeping, in: Le boom de mercenariat: defi ou fatalite?, Document de Damocles, Lyon

Bundesministerium für wirtschaftliche Zusammenarbeit und Entwicklung (BMZ) (2004): Zum Verhältnis von entwicklungspolitischen und militärischen Antworten auf neue sicherheitspolitische Herausforderungen, in: BMZ-Diskurs 1, Mai, Bonn

Eppler, E. (2002): Vom Gewaltmonopol zum Gewaltmarkt, Frankfurt/M. Holmquist, C. (2005): Private Security Companies. The Case for Regulation, in: SIPEI Policy Paper 9, Stockholm

Isenberg, D. (2004): A Fistful of Contractors: The Case for a Pragmatic Assessment of Private Military Companies in Iraq, in: BASIC, Research Report, September

Jäger, T./Kümmel, G. (2007): Private military and security companies, Wiesbaden

Leander, A. (2002): Global Ungovernance: Mercenaries, States and the Control over Violence, Copenhagen

Metz, S. (2002): Armed Conflict in the Twenty-First Century: The Information Revolution and Postmodern Warfare, Strategic Studies Institute, US-Army War College, April
Moller, B. (2002): The Political Economy of War. Privatisation and Commercialisation, COPRI Working Paper 16, Copenhagen

Pagliani, G. (2004): II mestiere della Guerra, Mailand

Petersohn, U. (2006): Die Nutzung privater Militärfirmen durch US-Streitkräfte und Bundeswehr, Berlin, SWP-Studie S 36, Dezember

Richards, A./Smith, H. (2007): Addressing the Role of private security companies within security sector reform programmes, Januar, London Schaller, C. (2005): Private Sicherheits- und Militärfirmen in bewaffneten Konflikten, SWP-Studie S 24, September, Berlin

Schreier, F./Caparini, M. (2005): Privatizing Security: Law, Practice and Governance of Private Military and Security Companies, Genf Singer, P. W. (2003): Corporate Warriors. The Rise of the Privatized Military Industry, Ithaka and London

The Center of Public Integrity (2004): Making a Killing. The Business of War, Washington

Uesseler, R. (2006): Krieg als Dienstleistung. Private Militärfirmen zerstören die Demokratie, Berlin

Lilly, D./Vaux, T./Seiple, C./Nakano, G./Van Brabrant Vaux, K. (2002): Humanitarian Action and Private Security Companies, London Wulf, H. (2005): Internationalisierung und Privatisierung von Krieg und Frieden, Baden-Baden 